\title{
Load balancing with multiple cloud services using PSO Techniques
}

\author{
V Priyatharsini ${ }^{1}$, R.Kalaivani ${ }^{2}$, Dr.C.Balasubramanian ${ }^{3}$ \\ ${ }^{1}$ PG Student, ${ }^{2}$ Assistant Professor, ${ }^{3} \mathrm{HOD}$ \\ Department of Computer Science and Engineering, \\ P.S.R Rengasamy college of Engineering for Women, \\ Sivakasi, Tamilnadu, \\ India
}

\begin{abstract}
There are various problems occur to choose the best path during critical circumstances. PSO is one of the heuristic technique which can be randomly choose a particle and observe the particle's position. It can be mainly focused on the rotation of the object. It mainly based on non - batch scheduling algorithms. In this paper, the cloud is the data center to secure user's individual cloudlet and focus on the security of the user. VM'S allocated based on the no. of cloudlets in the data center. It can be similar via amazon.in, salesforce.com \& flipkart.com etc. It can be used for real time application in many firms and maintain traffic control in metropolitan city. Many crucial problems can be solved using PSO in minimum time. It contains more performance better than random assignment and genetic scheduler algorithms.
\end{abstract}

Keywords : VM, PSO, cloudlet, data center, cloud computing, brokers

\section{INTRODUCTION}

Cloud computing is solitary of the innovative technology to pile their records in the warehouse. Each customer has their appropriate id and password. Each customer can simply extract their facts in the arrangement similar data mining. Ex: Gilt citation from the collieries.

\section{BACKGROUND \& RELATED WORKS}

Evacsys: A cloud Based Service for Emergency Evacuation [1], Cloud computing is an embryonic and advanced platform that offers outsourcing to enrich the scalability of computing and storage resources. In tragedy scenarios, cloud computing can be essentially applied to route massive bulks of automobiles to reach actual time and makes it safer to reach quick destination. We classify automobiles as either evacuee's Trucks or superior emergency vehicles, such as Ambulances, fire department trucks and police vehicles.

What Is the Most Efficient Way to Select Nearest Neighbor Candidates for Fast Approximate Nearest Neighbor Search [9], Approximate nearest neighbor search (ANNS) is a basic and vital technique used in numerous chores such as object gratitude. It comprises dual methods: picking nearest neighbor candidates and execution a brute-force exploration of these applicants. Only the former though has space for upgrading. In most existing procedures, it 


\section{Priyatharsini et al., Load balancing with multiple cloud services using PSO Techniques}

approaches the cosmos by quantization. It then computes all the spaces between the demand and all the quantized morals (e.g: clusters or bit sequences), and picks a static number of applicants local to the probe. The act of the technique is estimated based on correctness as a role of the number of applicants. In this red-top, we suggest a novel ANNS way that receipts into excuse costs in the collection manner. Whereas existing methods employ computationally luxurious performances such as relative sort and heap, the future method ensures not. This recognizes an ominously well-organized rifle.

A Heuristic Procedure for Designing a Distribution Network in a Supply Chain System Using Harmony Search Metaheuristic and Linear Programming [17], One of the best key glitches in supply chain controlling is the supply network design problematic system which comprises locating production plants and delivery barns and defining the best tactic for distributing the invention from the foliage to the warehouses and from the warehouses to the clienteles. Our zone is to select the optimal numbers, locations and capacities of plants and warehouses to open so that all client demand is fulfilled at tiniest total expenditures of the distribution network. We established a mixed integer programming model for the problematic and solved it by a heuristic process which contains 2 sub-procedures. We used harmony-search meta-heuristic as the key process and linear programming to solve transshipment tricky as a subroutine at any rehearsal of the key process.

Video Surveillance for Smart Phones with SMS Alert Using Background Subtraction Algorithm [4], This article presents design to advance surveillance requests based on the usage of the service oriented prototype, with android smart handsets as user terminals, permitting use vigorous composition and swelling the rigidity of the scheme. According to the outcome of poignant object recognition study on video schemes, the exertion of the entities is traced via video surveillance. The poignant object is recognized by means of the image subtraction way. The contextual image is subtracted from the forefront image. From that the poignant object is resultant. So the frame algorithm and the verge rate are planned to discovery the poignant image. Using frame process the moving frame is recognized. Then by the verge value the measure of the mount is known and traced. Hence the effort of the entity is known exactly.

Emergency Alert Systems over Private Network Using Message Broadcasting [10], SMS is a loftier tactic for spread of EAS mails i.e. distribution serious data during spare circumstances to defend lives and possessions of folks. Plentiful firms have merged with third-party suppliers to deliver efficient results. And also we define about safety issues and restrictions with this tactic. It proves that this progressively executed safety set-up does not accomplish its recognized requirements for bulky residents.

Accident alert using GPS technology \& automated traffic light control for ambulance [3], Currently the highest root of death due to street fates is not because of the effect of the fate on the individual but because of the absence of maintenance and sudden action after the incident. Accident detector is a trick which is meant to notice the occurrence of chance or shake over a boundary that may occur to an automobile during its gesture. Here accelerometer sensors the modification in acceleration began by the tremor or tilt. The detection of chance is done by the microcontroller after scrutiny the yield of accelerometer. When the accident is detected, the site is decoded from the crop of a GPS module and this file is send as a message to an emergency call center by using a GSM module. The marvelous rise in quantity of vehicles that seem on the roads have enlarged so much that it makes blocks and chaos everywhere. 
Real-Time Map Reduce Scheduling [16], In this paper, we discover the possibility of allowing the scheduling of varied hard and soft real-time Map Reduce claims. We chief present an tentative appraisal of the widespread Hadoop Map Reduce. Map Reduce middleware on the Amazon EC2 cloud. Our assessment reveals compromises between overall scheme throughput and implementation period predictability, as well as high spot a amount of types disturbing real-time scheduling, such as data placement, simultaneous users and master scheduling overhead. Based on our appraisal learning, we present a proper typical for taking real-time Map Reduce applications and the Hadoop platform. Using this typical, we express the offline development of real-time Map Reduce jobs on a heterogeneous dispersed Hadoop planning as a limitation satisfaction problematic and announce numerous search plans for the design. We suggest an improvement of Map Reduce's accomplishment exemplary and a variety of exploratory techniques for the online scheduling.

Implementation of Real Time Alert System over Cloud Computing [11], In recent years, cloud computing is widespread that can offer an exposed, flexible and reconfigurable platform for numerous supervisory tenders. Our organization knows a variation of trials that happen in the censored galaxy and alerts them a regulator center in actual time.

Weaather Montoring Station With Remote Radio Frequency Wireless Communications [14], Climate records is vigorous before affecting a flurry, exclusively dealings that unswervingly narrate to surroundings such as husbandry deeds. Conditions analyzer is engaged from the hotness and cosmological emission control etc.

Application of Firefly Algorithm in Job Shop Scheduling Problem for Minimization of Make span [8], Job scheduling problematic is a well-known scheduling problematic in which supreme of them are characterized into NP problematic because of its intricacy. Numerous academics intended to explain the problematic by smearing countless optimization methods. While using traditional approaches they pragmatic vast strain in resolving complex problems.

Natural disasters their impact on electricity supply [15], The safety of energy supply has developed a main anxiety worldwide, given contemporary society's resilient necessity on its tolerable transport. Hurries in energy beliefs, dogmatic battles, battles and typical misfortunes unswervingly portend potency supply and vital plan anxieties are presence executed as nations look at means to shield themselves.

Da symmetric Mapping of Building Stocks within HAZUS-FL [2], The accuracy of surfeit damage models is contingent on the joining between the spots of the GBS and flood soaks. To confirm that FL relics pertinent to wide viewers, procedures are anticipated to enrich the exactness of these issues in the FL archetypal which do not need further exhaustive building datasets or vary the prevailing FL software code. Refining the GBS illustration by smearing consistency to the GBS would increase the exactitude of the FL model evaluations. This hypothesis shows the probability of dissymmetric GBS by smearing plot asylum facts to align the GBS with reputable land to inflate the correctness of FL replicas. These stuffs are ultimate noticeable in zones with partial flood or little density growth. CBGs feeling severe submerging or great density growth exhibited restricted damage adjustments related to the recent FL building format. 


\section{Priyatharsini et al., Load balancing with multiple cloud services using PSO Techniques}

Surveillance of Object Motion Detection and Alert Using Android [6], Cinematic Observation structures have swell their necessities of potency in demand to permit different employers to viewer the system picking different QoS reliant on the arrangement status and to access conscious and illustrious audiovisual from different localizations, This observation constructed service arrangement provides welfare for specific residence which counsels the worker by conveyance alert messages. We discuss a innovative process, Image subtraction procedure that seizures the image routinely and is protected in the attendant. The protected image is sent as a cautious to the operator portable using Google Cloud Messaging.

Remote monitoring using an induction loop [20], An electromagnetic spur coil is recycled to handover gestures from a throb and gulp curtain to a archetypal National Health Service hearing aid to plane isolated perceiving.

Development of a Remote Automatic Weather Station with a PC- based Data Logger [7], This red-top assistances the advance of a archetype climate station to aggregate the successive climate records: The climate location which has been cautious for reserved process accomplishes reflex or unmanned scopes of survive accounts and bears it wirelessly to a PC for labeling and confirmation via capitals of a graphical user interface. The detached posting is mechanical by astral vigor concluded a battery which also supplies resistor for a action of the outline. Faltering results display that the unemotional records are fairly consistent with those grew via comparable climate quantity tactics.

Automated Weather Sensor Quality Control [13], In this rag, we examine the solicitation of records mining to surviving practices for superiority regulator on climate sensor annotations. Specifically we adjust the prevalent Barnes 3-D interruption technique to use time sequence reserve pretty than spatial remoteness to evolution a reinforced procedure that uses interpretations from alike stations built on recent and antique explanations for interruption. We reveal that this innovative process revelations less classical blunder than the Barnes Spatial interpolation based manner. Our tactic displays enhanced recital as indicated by mean squared error abridged via roughly partial for expected ethics contrasted with reported ethics.

Nearest Neighbors Using Compact Sparse Codes [5], In the former rare years, there has been a enormous growth in the expanse of customer created data in the web. On the solitary, accessibility of such enormous archives offers wonderful chances for creating innovative claims. On the other hand, consolidating such enormous data, so that it is purely and prompt reachable is a test. In this adoration, a core portion in plentiful processes that exertion in retrieving such immense records is that of ANN in which the area is to recover a subgroup of a dataset that is maximum analogous to a probe.

Comparative Study of Firefly Algorithm and Particle Swarm Optimization for Noisy Non- Linear Optimization Problems [12], Linear Optimization Snags, There are numerous deafening non-linear exact optimization glitches that can be efficiently cracked by Meta heuristic Processes. These are iterative pursuit practices that capably make the investigation and corruption in the key space, targeting to capably discovery proximate optimal keys. Since the result planetary in a definite state, some replicas contain worldwide peak and various resident optima. In this context, dual forms of meta-heuristics called PSO and Firefly processes were formulated to discovery finest keys of boisterous nonlinear unceasing scientific models. The effects of this testing were examined and related to the finest keys found so far on the root of mean of performance period to congregate to the best. The Firefly process seems to achieve improved for advanced stages of blare. 
Firefly Algorithms for Multimodal Optimization [18], Nature-inspired processes are amongst the greatest prevailing processes for optimization. This rag intends to offer a complete depiction of an innovative FA for multimodal optimization requests. We will equate the future firefly process with former meta heuristic systems such as PSO. Imitations and outcomes specify that the future firefly process is higher to prevailing Meta heuristic systems. Lastly we will deliberate its requests and allegations for additional study.

Real-Time Virtual Machines [19], This broadside tackles the delinquent of ensuring suitable suitability promises to simultaneous uses consecutively in a virtualized Effective Scheme. Initial tentative outcomes are offered, emphasizing how the proper use of engrained simultaneous development policies may be active in fronting with this interesting dispute.

\section{PROPOSED SYSTEM}

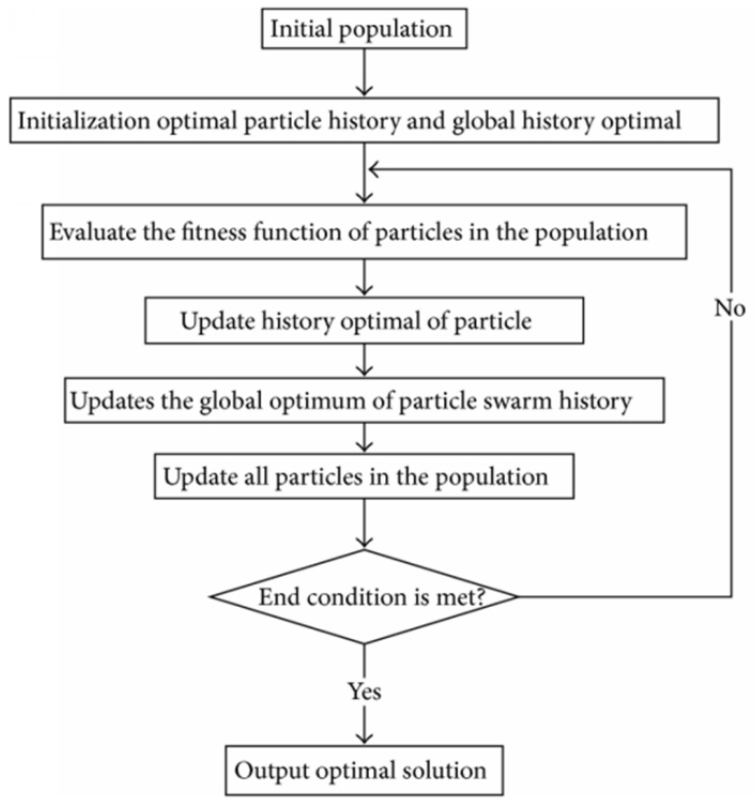

Fig 1: System Architecture

PSO represents one of the problems solving method used for many real time applications. It contains non-batch scheduling technique. It contains quantity of the customers based on the no. of. VM allowed in datacenters. Each customer contains various format of cloudlet which will store in DC need privacy and security upgrade day by day. It contains very fast $\&$ operate easily and provide accurate result contain in it.

\section{Modules}

$>$ Data center creation

$>$ Broker Creation 
V Priyatharsini et al., Load balancing with multiple cloud services using PSO Techniques

$>$ Virtual Machine Creation

$>$ Cloudlet Creation

Allocate cloudlet to Virtual Machine

\section{Modules Description}

\section{Data Center Creation}

In olden days human store their data in huge area and not easy to secure their data in easy manner. They need reduced cost and compact format of data storage. So, they move on to the data centers in the cloud. Avoid data loss and enrich the data size \& security is easy. Each datacenter contains datacenter name, datacenter id, host name, Ram etc.

\section{Broker Creation}

Broker is a third party which acts as a legitimate between the two parties. They can satisfy both party needs and maintain their complaints. The amount of Brokers based on the number of data center and VM. It can satisfy the terms and conditions of VM and datacenter.

\section{Virtual Machine Creation}

VM which contains cloudlets of individual user can be store and executed for future use. The cloudlet contains various user names and contains individual id for user, host and broker etc for future use. Reduced cost and maintained of each users.

\section{Cloudlet Creation}

The cloudlet means numerous amounts of files which can be stored in VM via broker id. The cloudlets can be easily expanding their bandwidth of the data. The data can be stored in the time period can be stored and they can be maintain for future use.

\section{Allocate Cloudlet to VM}

In this module some cloudlets can store their data in the VM. Numerous cloudlets can be allotted to VM dynamically. These types of application can be implemented in software production and testing process named outsourcing.

\section{Conclusion \& Future Enhancement}

This article recommended that an appropriate excellent for secure critical problems. The PSO was extraordinary equated with QPSO in quicker convergence. In this tactics, entirely calculated among different methods were stated using the equal amount of repetitions \& residents scope. 


\section{Implementation}

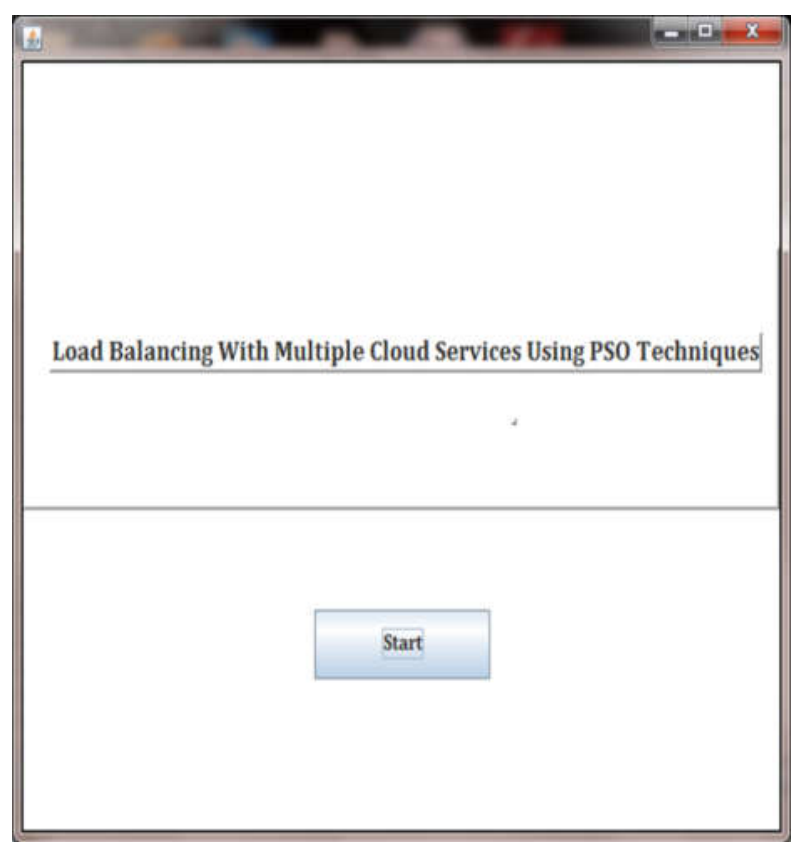

Fig 2: Home page creation

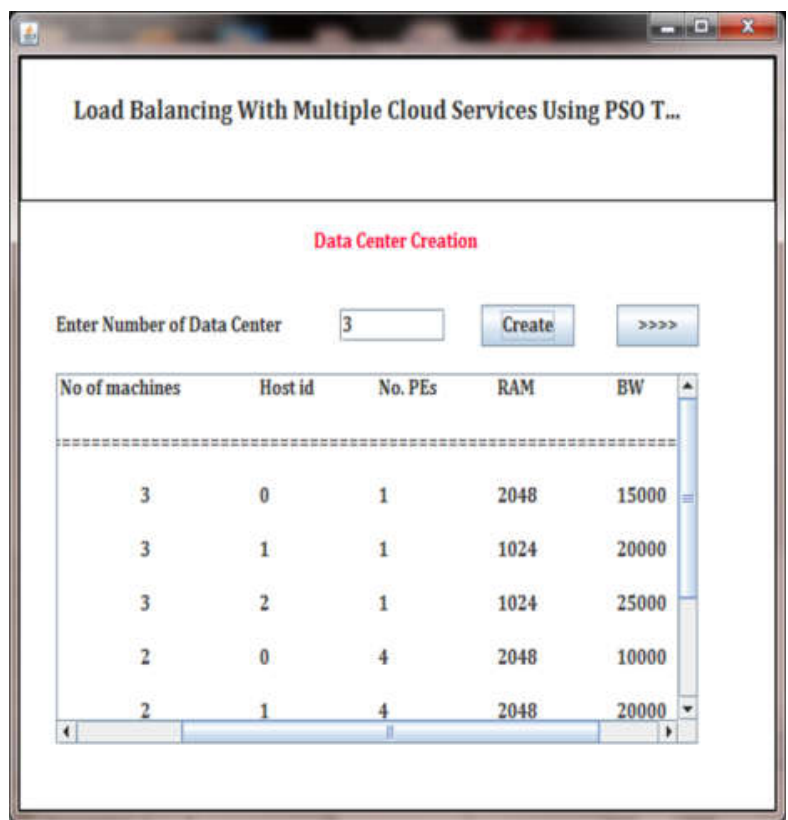

Fig 3: Data Center Creation

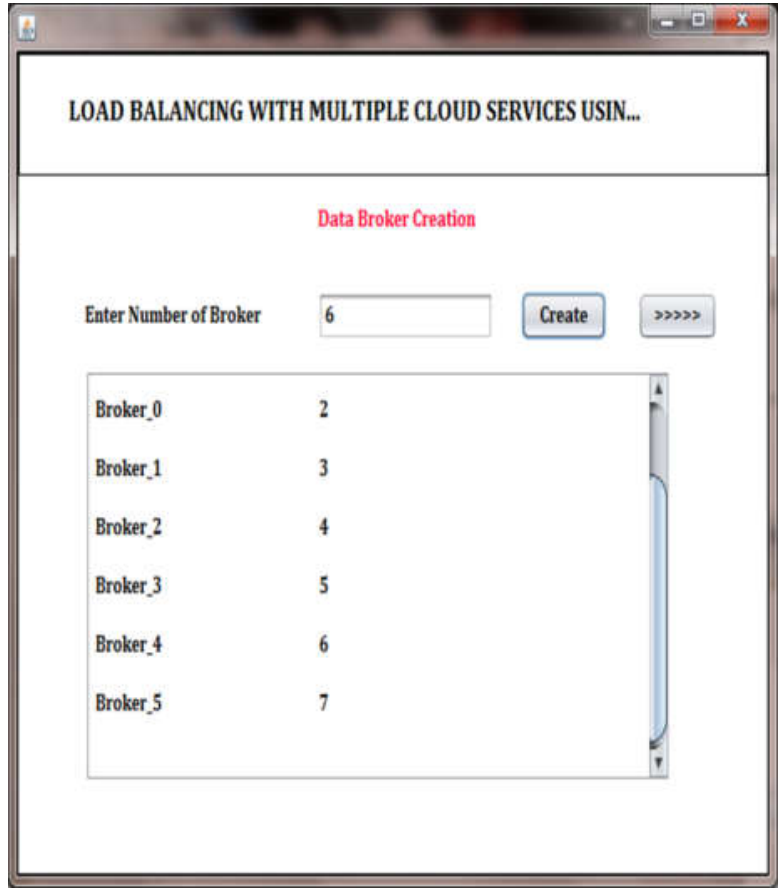

Fig 4: Broker Creation

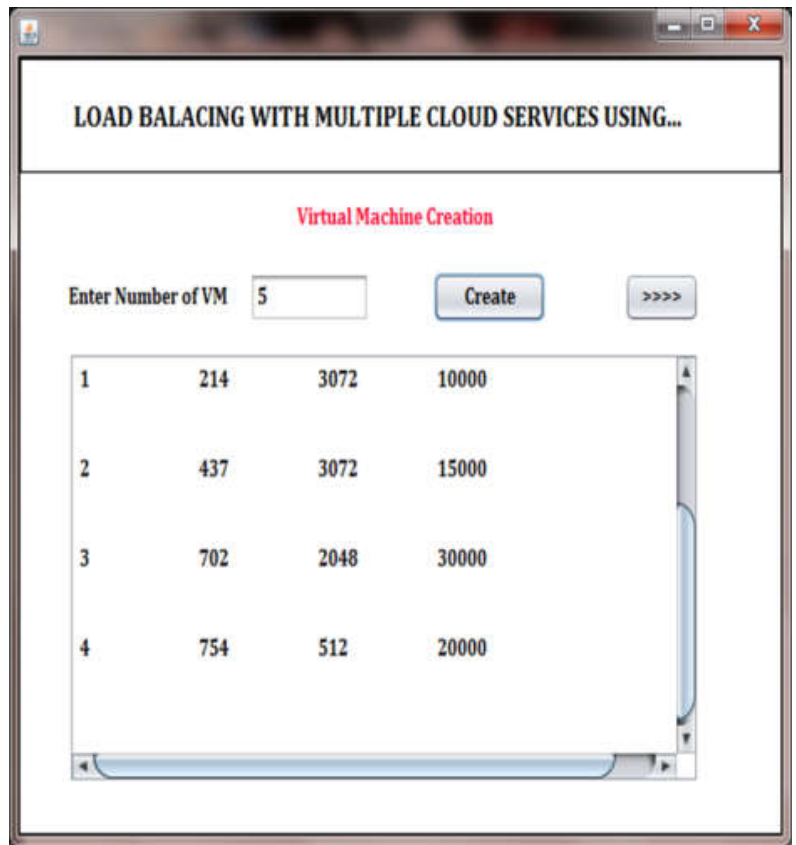

Fig 5: VM Creation

\section{REFERENCES}

[1] Osman Khalid, Muhammad Shahid Usman Khan, Ying Huang, Samee U .Khan, Albert Zomaya, "EvacSys : A Cloud Based Service for Emergency Evacuation,” IEEE International conference 2016; WWW.COMPUTER.ORG 
[2] Brian Patrick McDonald,” Dasymetric Mapping of Building Stocks within HAZUS-FL”, IEEE May 2016.

[3] Dhanoj M, Ashwin Prem, Bibi Mohan, Ashwin Prem," Accident alert using GPS technology \& automated traffic light control for ambulance", International Conference on RECENT TRENDS IN ENGINEERING AND MANAGEMENT, IEEE Vol. 4, Issue 1, Jan 2015, www.iosrjournals.org.

[4] S. Kannan, B. Sundar Raj," Video Surveillance for Smart Phones with SMS Alert Using Background Subtraction Algorithm”, DOI: 10.15680/IJIRCCE.2015. 0309206 8228, Vol. 3, Issue 9, Sept 2015.

[5] Anoop Cherian, "Nearest Neighbors Using Compact Sparse Codes", Proceedings of the 31 st International Conference on Machine Learning, Beijing, China, JMLR:W\&CP vol 32, IEEE 2014.

[6] S. Shahid Ali Basha, T. Senthil Kumar," Surveillance of Object Motion Detection and Alert Using Android", Volume 2, Issue 1, 2014.

[7] Roneel, V. Sharan, "Development of a Remote Automatic Weather Station with a PC-based Data Logger", IEEE 2014

[8] K.C. Udaiyakumar, M. Chandrasekaran," Application of Firefly Algorithm in Job Shop Scheduling Problem for Minimization of Make span”, doi: 10.1016/j.proeng.2014.12.333 2014.

[9] Masakazu Iwamura, Tomokazu Sato and Koichi Kise, "What Is the Most Efficient Way to Select Nearest Neighbor Candidates for Fast Approximate Nearest Neighbor Search?”, IEEE International Conference on Computer Vision, DOI 10.1109/ICCV.2013.439 2013.

[10] B. Venkata Phani Raja Rao, K.R.K. Sateesh, Emergency Alert Systems over Private Network Using Message Broadcasting", International Journal of Advanced Research in Computer Science and Software Engineering Volume 3, Issue 11, November 2013.

[11] Jaeseok Shim and Yujin Lim, " Implementation of Real Time Alert System over Cloud Computing", IEEE 2013. www.aaai.org

[12] Saibal K. Pal, C.S Rai, Amrit Pal Singh, "Comparative Study of Firefly Algorithm and Particle Swarm Optimization for Noisy Non- Linear Optimization Problems", Intelligent Systems and Applications, 10, 50-57, DOI: 10.5815/ijisa.2012.10.06, sept 2012.

[13] Douglas Galarus, Rafal Angryk and John Sheppard,” Automated Weather Sensor Quality Control”, IEEE 2012, 
V Priyatharsini et al., Load balancing with multiple cloud services using PSO Techniques

[14] Ishwanto, Helman Muhammad," Weather Monitoring Station with Remote Radio Frequency Wireless Communictions", International Journal of Embedded Systems and Applications (IJESA) Vol.2, No.3, Sept 2012.

[15] "Natural disasters their impact on electricity supply", Digital Object Identifier 10.1109/MPE.2010.939922, IEEE power and engine magazine, April 2011, www.rhmintl.com.

[16] Linh T. X. Phan, Zhuoyao Zhang, Boon Thau Loo, Insup Lee” Real-Time Map Reduce Scheduling”, IEEE Jan 2010, http://repository.upenn.edu/cis_reports/942.

[17] M. Rahimi, A. Hemmati, M. Bakhshayeshi Baygi, S. M. Mirpourian, A. A. Jaafari, "A Heuristic Procedure for Designing a Distribution Network in a Supply Chain System Using Harmony Search Meta-heuristic and Linear Programming," IEEE 2010.

[18] Xin-She Yang,"Firefly Algorithms for Multimodal Optimization”, O. Watanabe and T. Zeugmann (Eds.): SAGA 2009, LNCS 5792, pp. 169-178, 2009.

[19] Tommaso Cucinotta, Gaetano Anastasi, Luca Abeni," Real-Time Virtual Machines", IEEE 2008; www.planet lab.org.

[20] M. J. Harrison and E. J. Slater, ’Remote monitoring using an induction loop”, IEEE 1986,. 